\title{
Transtorno de ansiedade generalizada entre estudantes de cursos de pré-vestibular
}

Generalized anxiety disorder among university-entrance preparation course students

DOI: $10.1590 / 0047-2085000000277$

J Bras Psiquiatr. 2020;69(3):179-86

Where you read:

Received in: Apr/4/2020. Approved in: Apr/26/2020

Should read:

Received in: Dec/12/2019. Approved in: Apr/4/2020

DOI: 10.1590/0047-2085000000317 